\title{
Note on Modernization
}

When quoting early modern editions of texts in English, I have supplied the modern typographical equivalents of certain letters $(v$, $v v, u$ and $f$ ). Titles of early modern editions have been shortened in the bibliography but are reproduced liberally in the notes. For the sake of consistency, titles, punctuation and publishing information for these works have all been keyed to the second edition of Pollard's and Redgrave's Short-Title Catalogue of Books Printed in England, Scotland and Ireland, 1475-1640. 

Let us dismiss the accidental; for we have sufficiently determined its nature.

-Aristotle, Metaphysics 1027b 
